\title{
Analysis and simulation of high-power LED array with microchannel heat sink
}

\author{
Xin Zhang $\cdot$ Ru-Chun Li $\cdot$ Qi Zheng
}

Received: 28 January 2013/Accepted: 13 May 2013/Published online: 14 June 2013

(C) Shanghai University and Springer-Verlag Berlin Heidelberg 2013

\begin{abstract}
The high power light emitting diode (LED) array integrated with the microchannel heat sink is designed in this paper, and then optimal analysis and simulation have been carried out. According to the theory of heat transfer and fluid mechanics, the calculation of the thermal resistance for the microchannel heat sink is obtained, and the thermal resistance is minimized. Finally the simulation with FLUENT software is developed to verify the theoretical analysis. Established analysis and simulation show that the width of the cooling channel is $0.1 \mathrm{~mm}$, and the cooling water flow rate is $1 \mathrm{~m} / \mathrm{s}$. On the other hand, the system acquires the best heat dissipation effect, and the minimum of thermal resistance is $0.019 \mathrm{~W} /{ }^{\circ} \mathrm{C}$.
\end{abstract}

Keywords High-power LED array - Microchannel heat sink - Thermal resistance - Analytical solution ·

Optimized simulation

\section{Nomenclature}

$l, w$ and $z \quad$ Heat sink length, width and height (m)

$l, w_{\mathrm{c}}$ and $z_{\mathrm{c}}$ Channel length, width and height $(\mathrm{m})$

$w_{\mathrm{w}} \quad$ Fin width (m)

$n \quad$ Number of channels

A The total heat sink area

$d \quad$ Hydraulic diameter (m)

$p \quad$ Cross-sectional perimeter

$h \quad$ Heat transfer coefficient $\left(\mathrm{W} /\left(\mathrm{m}^{2} \cdot{ }^{\circ} \mathrm{C}\right)\right)$

$\lambda \quad$ Fluid thermal conductivity $\left(\mathrm{W} /\left(\mathrm{m} \cdot{ }^{\circ} \mathrm{C}\right)\right)$

$\lambda_{\text {fin }} \quad$ Thermal conductivity $\left(\mathrm{W} /\left(\mathrm{m} \cdot{ }^{\circ} \mathrm{C}\right)\right)$

$\mathrm{Nu} \quad$ Nusselt number

X. Zhang $(\bowtie) \cdot$ R.-C. Li $\cdot$ Q. Zheng

College of Information Engineering, Zhejiang University of

Technology, Hangzhou 310023, People's Republic of China

e-mail: 18767123506@163.com

$\begin{array}{ll}P r & \text { Prandtl number } \\ R e & \text { Reynolds number } \\ R & \text { Thermal resistance }\left({ }^{\circ} \mathrm{C} / \mathrm{W}\right) \\ \mathrm{Q} & \text { The dissipation power }(\mathrm{W}) \\ \mu & \text { The fluid dynamic viscosity in the } \\ & \text { qualitative temperature }(\mathrm{kg} /(\mathrm{m} \cdot \mathrm{s})) \\ \mu_{\mathrm{w}} & \text { The fluid dynamic viscosity in the tube wall } \\ & \text { temperature }(\mathrm{kg} /(\mathrm{m} \cdot \mathrm{s})) \\ \Delta t & \text { The maximum temperature difference } \\ & \text { between the source and coolant }\left({ }^{\circ} \mathrm{C}\right) \\ N u_{\infty} & \text { Nusselt number for fully developed flow } \\ f & \text { The volume flow velocity }\left(\mathrm{m}^{3} / \mathrm{s}\right) \\ \rho & \text { Fluid density }\left(\mathrm{kg} / \mathrm{m}^{3}\right) \\ C_{\rho} & \text { The fluid specific heat } \\ v & \text { The fluid average flow velocity }(\mathrm{m} / \mathrm{s}) \\ \Delta p & \text { The entrance pressure }(\mathrm{psi})\end{array}$

\section{Introduction}

Maximizing power output has been the main task for the development of high-power light emitting diode (LED) array applications such as illumination. Due to the performance improvement of the diode, the output power is likely to go up to $5-10 \mathrm{~W}$ from a single diode. The heat flux of the high-power LED array has reached $100 \mathrm{~W} / \mathrm{cm}^{2}$ [1], however more than half of the power is to be dissipated in form of waste heat within limited area. These will cause that the chip temperature goes up, and the emitted photons quantity is reduced. The efficiency of light is decreased, which will also affect the quality of light source and have a reduction of service life. Consequently, improved thermal management techniques are required for the LED array [2]. 
The concept of micro-channel heat sink is firstly introduced by Tuckerman and Pease in 1981 [1]. Its superior performances include compact solid structure, high cooling efficiency, and it is easy to integrate with the high-power optoelectronic device and so on. It is a new idea that the microchannel technology is applied in the cooling system of high-power LED array. This design scheme combines the theoretical analysis with simulation, which can effectively decrease the heat dissipation. As long as there is an appropriate coolant, it can step into commercialization stage gradually uniting with the micro-pump technology and dropping the cost.

\section{Structure design}

As the external heat sink for the LED array, the microchannel structure design is very critical, directly affecting the whole cooling capacity of the system. Conveniently, the structure of straight fin with the rectangular cross section is adopted in the design of microchannel [3] (see Fig. 1). The high-power LED array attached with the microchannel heat sink is shown in Fig. 2. It has five rows of LEDs, which are arranged in parallel in each row. The dies are spaced about $6 \mathrm{~mm}$ center-to-center distance across the

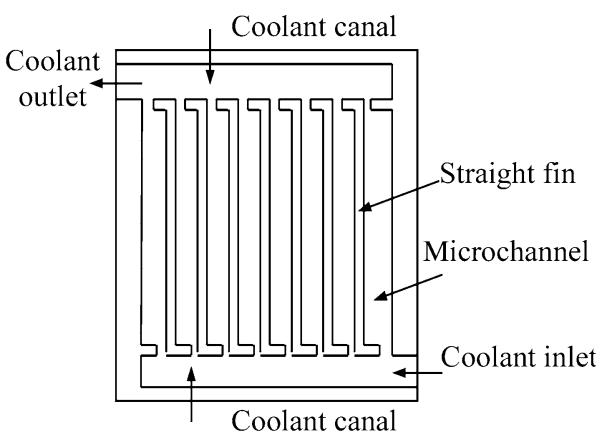

Fig. 1 Schematic structure of the microchannel heat sink with straight fins (horizontal section)

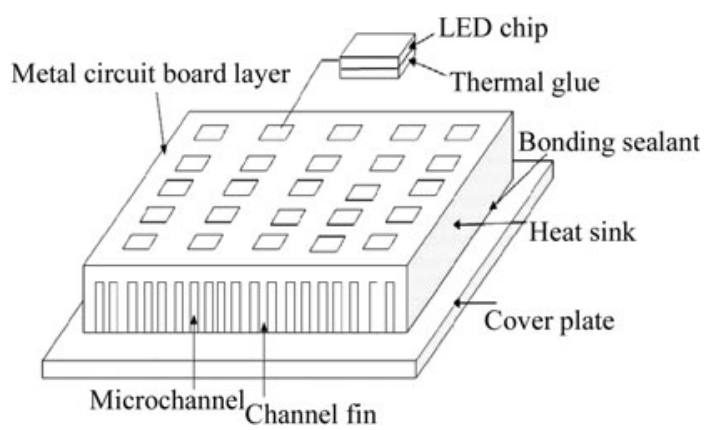

Fig. 2 Schematic view of high-power LED array integrated with microchannel heat sink
Table 1 Material parameters and size

\begin{tabular}{llll}
\hline Component & Length/mm & Width $/ \mathrm{mm}$ & Height $/ \mathrm{mm}$ \\
\hline LED chip & 3 & 3 & 0.25 \\
Glue & 3 & 3 & 0.25 \\
Heat sink & 40 & 33 & 2 \\
Cover plate & 46 & 39 & 0.5 \\
\hline
\end{tabular}

length of the heat sink. LED dies are solder-bonded to the metal circuit board layer, which is directly connected with the microchannel heat sink. A cover plate is bonded to the substrate to confine the coolant in the channels. The system's major geometric size is shown in Table 1.

\section{Calculation of thermal resistance}

Thermal resistance is a major parameter characterizing the system thermal performance. The smaller is the thermal resistance, the better performance of cooling system. The thermal resistance in this design is comprised of three parts. $R=R_{\text {cond }}+R_{\text {conv }}+R_{\text {heat }}$, where $R_{\text {cond }}$ is the heat conduction from the circuits through the substrate and heat sink interface; $R_{\text {conv }}$ is the heat convection from the heat sink to the coolant fluid; $R_{\text {heat }}$ is the thermal resistance caused by the heat of coolant absorption [4].

Referring to the thermal resistance definition $(R=\Delta t / Q)$ [5], the Fourier heat conduction law and the Newton cooling formula [6], the sum of $R_{\text {cond }}$ and $R_{\text {conv }}$ can be obtained:

$R_{\text {cond }}+R_{\text {conv }}=\frac{z_{\mathrm{t}}}{\lambda_{\text {fin }} l w}+\frac{1}{h A_{\mathrm{s}}}$,

where the channel inner surface area $A_{\mathrm{s}}=\alpha A=\alpha l w . \alpha$ is the ratio of total surface area of channel walls in contact with fluid to the area of circuit board layer. $z_{t}$ is the height of the channel top to heat sink surface. $R_{\text {cond }}$ can be made very small by locating the heat exchanger (containing the flowing coolant) very near to the heat source. $R_{\text {conv }}$ and $R_{\text {heat }}$ will be the dominant consideration in high-performance heat sink design.

$d$ is the hydraulic diameter of the channel, defined as $d=4$ (cross-sectional area)/(perimeter $p$ ). For high-aspect ratio rectangular channels, when $w_{\mathrm{c}}$ is largely smaller than $z_{\mathrm{c}}, d$ is equal to twice as the channel width, expressed as

$d=4 \times \frac{w_{\mathrm{c}} z_{\mathrm{c}}}{2\left(w_{\mathrm{c}}+z_{\mathrm{c}}\right)} \approx 2 w_{\mathrm{c}}$.

The fluid flow involved in this paper is laminar flow, and as for the laminar flow, Nusselt number is

$N u=1.86(\operatorname{RePr})^{\frac{1}{3}}\left(\frac{d}{l}\right)^{\frac{1}{3}}\left(\frac{\mu}{\mu_{\mathrm{w}}}\right)^{0.14} \approx N u_{\infty}$, 
where $N u_{\infty}$ is a constant situated between 3 and 9, whose precise number is dependent on the shape of the channel [7].

The cooling fin efficiency $\eta$ is the ratio of the actual heat dissipating capacity to the heat dissipating capacity assumed the fin surface in rib base temperature [8]. On the same cross section of rectangular fin, $\eta$ is:

$\eta=\frac{\tan H}{H}$

$H=\left(\frac{2 h}{w_{\mathrm{w}} \lambda_{\mathrm{fin}}}\right)^{\frac{1}{2}} z_{\mathrm{c}}=\left(\frac{N u_{\infty} \lambda}{\lambda_{\text {fin }}}\right)^{\frac{1}{2}} \frac{w_{\mathrm{c}}+z_{\mathrm{c}}}{2\left(w_{\mathrm{c}} z_{\mathrm{c}}\right)^{\frac{1}{2}}} \alpha$.

From (4), it can be observed that $\eta$ is the monotonically decreasing function of $H$, which means $w_{\mathrm{c}}=w_{w}$. There is a maximum for $\eta$.

The thermal resistance produced by heat absorption of coolant is defined as

$R_{\text {heat }}=\frac{1}{c_{\rho} \rho f}$.

According to the thermodynamics formula [9], the coolant fluid velocity in the channel is $v=w_{\mathrm{c}}^{2} \Delta p / 12 \mu \mathrm{l}$, and the total coolant unit volume flow rate is $f=$ $n\left(\left(w_{\mathrm{c}} z_{\mathrm{c}}\right) v\right)=1 / 2 v w w_{\mathrm{c}} \alpha$. The sum of $R_{\mathrm{conv}}$ and $R_{\text {heat }}$ can be simplified into:

$R=R_{\mathrm{conv}}+R_{\text {heat }}=\frac{2}{\lambda N u_{\infty} l w}\left(w_{\mathrm{c}} \alpha^{-1} \eta^{-1}\right)+\frac{24 \mu l}{\rho C_{\rho} \Delta p w \alpha w_{\mathrm{c}}^{3}}$.

\subsection{Theoretical optimization of the channel width}

The size of the heat sink is fixed, and the fin takes the same width as the channel. Then the channel number is $n=\left(\left(w-w_{\mathrm{c}}\right) / 2 w_{\mathrm{c}}\right)$. Ignoring the heat conduction at the top and bottom of the heat sink channel,

$\alpha=\frac{2 n l z_{\mathrm{c}}}{n l\left(w_{\mathrm{c}}+w_{\mathrm{w}}\right)}=\frac{2 z_{\mathrm{c}}}{\left(w_{\mathrm{c}}+w_{\mathrm{w}}\right)}=\frac{z_{\mathrm{c}}}{w_{\mathrm{c}}} \Rightarrow z_{\mathrm{c}}=\alpha w_{\mathrm{c}}$.

For a practical design, referring to Eq. (5) and setting $\alpha=\lambda_{\text {fin }} /(N u-\lambda)^{1 / 2}$, we have $\eta=0.76$. Equation (7) is considered as a function of $R$ and $w_{\mathrm{c}}$ to solve its extreme value and make it equal to zero. Then the optimum solution is got

$w_{\mathrm{c}}=2.29 \sqrt[4]{\frac{\mu \lambda l^{2} N u_{\infty}}{\rho C_{\rho} \Delta p}}$.

Setting the density of the coolant $\rho=1 \mathrm{~g} / \mathrm{cm}^{3}$, the volumetric heat capacity $C_{\rho}=4.2 \mathrm{~J} /\left(\mathrm{g} \cdot{ }^{\circ} \mathrm{C}\right)$, the dynamic viscosity $\mu=1.01 \times 10^{-3} \mathrm{~Pa} \cdot \mathrm{s}$, the fluid thermal conductivity $\lambda=0.5 \mathrm{~W} /\left(\mathrm{m} \cdot{ }^{\circ} \mathrm{C}\right), \quad N u_{\infty}=6, \quad R e=730$, and the entrance pressure of $30 \mathrm{psi}$. These are all substituted into Eq. (9), and then, the most ideal channel width is obtained $w_{\mathrm{c}}=0.1 \mathrm{~mm}$.

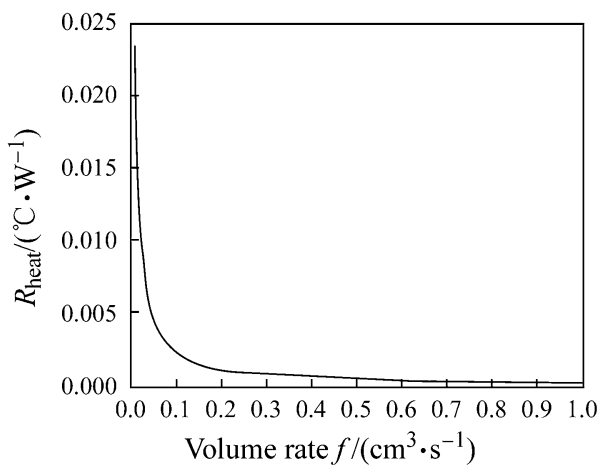

Fig. 3 Relationship curve of thermal resistance and cooling fluid velocity

\subsection{Theoretical optimization of the fluid velocity}

The cooling fluid flow rate is only relevant to $R_{\text {heat }}$, the curve of Eq. (6) is drawn in Fig. 3 with MATLAB.

In Fig. $3, R_{\text {heat }}$ rapidly decreases with the increase of $f$. After $f \gg 1 \times 10^{-7} \mathrm{~m}^{3} / \mathrm{s}=0.1 \mathrm{~cm}^{3} / \mathrm{s}, R_{\text {heat }}$ have little change. However, the coolant flow rate cannot unlimitedly increase. The greater the flow velocity, the greater the pressure drop. Synthesizing the above factors, $f=1 \times 10^{-7} \mathrm{~m}^{3} / \mathrm{s}$ is taken as the best coolant flow rate.

\section{Simulation analysis}

Detailed analysis on the flow field and heat transfer were performed with a computational fluid dynamics (CFD) software FLUENT to optimize the heat sink [10]. The best thermal performance of the high-power LED array is achieved, and the correctness of the theoretical analysis is verified.

Only dies are established without the phosphor and resin in the modeling. The simulation of heat sink is subject to several constraints: (i) Each one of the LED heat source is uniform and plane with a power consumption of $9 \mathrm{~W}$; (ii) The heat flux density $5 \times 10^{9} \mathrm{~W} / \mathrm{m}^{3}$ calculated by the thermodynamics formula has been imposed on the chip; (iii) The substrate is cooled through the heat conduction and the heat convection, and the coefficient of convective heat transfer is $6 \mathrm{~W} /\left(\mathrm{m}^{2} \cdot{ }^{\circ} \mathrm{C}\right)$ [11]; (iv) The coolant is deionized water with uniform inlet velocity, and the way of the fluid flow is laminar flow; (v) The environment temperature is $25^{\circ} \mathrm{C}$, which is the same as the initial fluid temperature; (vi) The thermal radiation effect is ignored, and the material is assumed that it is homogeneous, isotropic and continuous medium [12].

\subsection{Optimize simulation of the channel width}

The thermal performance of the high-power LED array with microchannel heat sink is simulated. The rectangular 
cross section is applied in the simulation, and the channel widths is $1,0.5,0.15,0.1$ and $0.08 \mathrm{~mm}$, respectively. The inlet fluid velocity is $1 \mathrm{~m} / \mathrm{s}$ when the height of the channel is fixed assuming that the chip power consumption is all converted into heat.

Figure 4 shows the temperature distribution of the LED array surfaces in the direction of the flow when the channel widths take on the values $1,0.1$ and $0.08 \mathrm{~mm}$. It can be seen that the temperature in upstream is significantly less than that

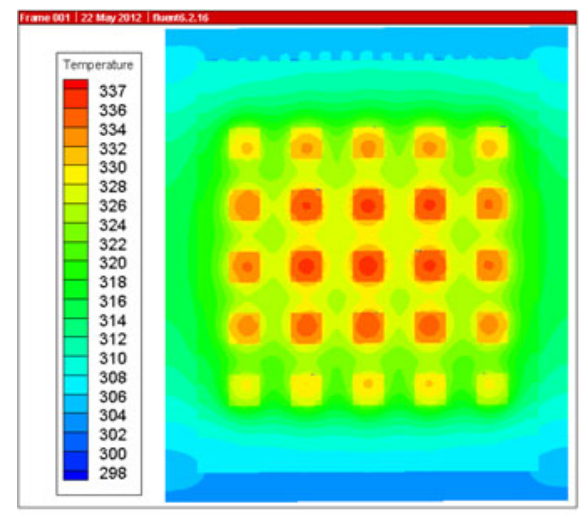

(a) Channel width: $1 \mathrm{~mm}$

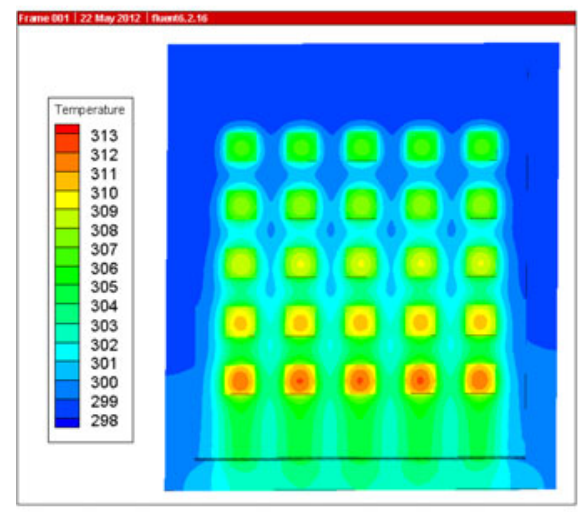

(b) Channel width:0.1mm

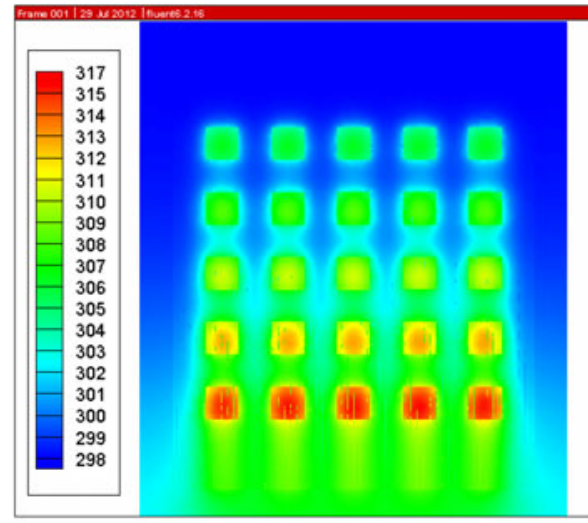

(c) Channel width:0.08mm

Fig. 4 Temperature distribution nephogram on the surface of the microchannel heat sink (Unit: K)

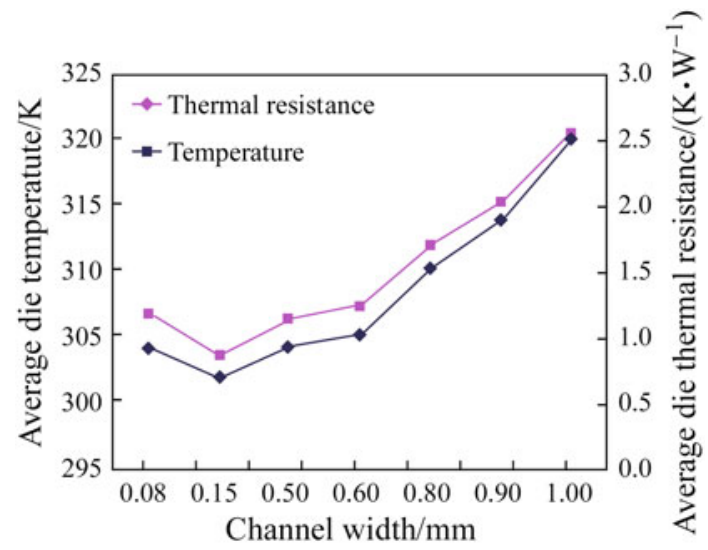

Fig. 5 Average temperature and thermal resistance versus channel width $(v=1 \mathrm{~m} / \mathrm{s})$

in downstream along the direction of the flow. In transverse direction of the flow, the temperature of chips in central area is higher than that in the edge. The main reasons of this phenomenon are: (i) The heat in the edge of the LED array can be dissipated from the heat sink side. However the temperature of the center LED chips is affected seriously due to the heat generated by other surrounding chips, which prevented the heat dissipation; (ii) The heat dissipation of the fluid in downstream performs slightly worse after preheating; (iii) The coolant velocity on the flow boundary is slower than that in the center of the coolant.

Figure 5 shows the curve of the average die temperature with the change of the channel width. The smaller is the width of the channel, the greater the cooling area, the higher the cooling efficiency. However the cooling fluid dynamic viscosity increases with the decrease of the channel width, which results in slower velocity and influencing the heat dissipation [13]. When $w_{\mathrm{c}}=0.1 \mathrm{~mm}$, the average temperature on the heat sink surface reaches the minimum, and the cooling performance is improved.

\subsection{Optimize simulation of the fluid velocity}

Under the condition of the same heat dissipation, the flow rates are respectively taken as $0.5,1,1.5,2 \mathrm{~m} / \mathrm{s}$. The influence of the microchannel heat sink temperature distribution caused by the cooling water flow is analyzed. Figure 6 shows the average temperature of the high-power LED array microchannel heat sink with different fluid velocities.

It can be seen from Fig. 6 that the decrease trend of the temperature of the microchannel heat sink with different channel widths is consistent. The trend of the temperature variation obviously reduced when $v>1 \mathrm{~m} / \mathrm{s}$, hence the best fluid velocity is $v=1 \mathrm{~m} / \mathrm{s}$. It is $f=1 \times 10^{-7} \mathrm{~m}^{3} / \mathrm{s}$ when converted to volume flow rate. Figure 7 clearly 


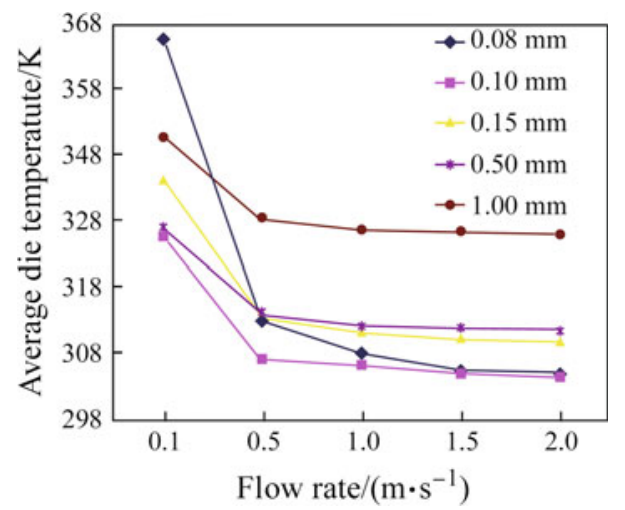

Fig. 6 Average die temperature versus flow rate

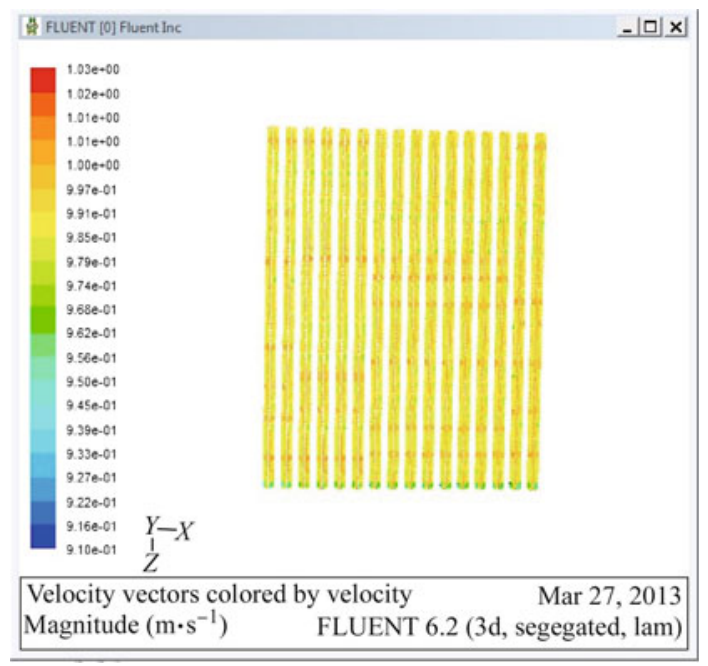

Fig. 7 Coolant velocity vector field $\left(w_{\mathrm{c}}=1 \mathrm{~mm}, v=1 \mathrm{~m} / \mathrm{s}\right)$

shows that the flow condition of the laminar state in the channel. When the fluid velocity is $1 \mathrm{~m} / \mathrm{s}$, the channel width is $1 \mathrm{~mm}$.

\section{Conclusions}

The cooling efficiency of the microchannel heat sink can be improved by increasing the channel quantity and reducing the channel diameter. However, the cooling fluid dynamic viscosity will increase with the decrease of the channel width. Considering a compromise, there is a best channel width $w_{\mathrm{c}}=0.1 \mathrm{~mm}$ achieved through the calculation and simulation.
The relationship between the heat dissipation capacity and the flow velocity are investigated. With the increase of the flow velocity, the average die temperature decreases. It will also cause the increase of the cooling fluid dynamic viscosity. The best flow rate $v=1 \mathrm{~m} / \mathrm{s}$ should be obtained by the calculation and simulation. Finally, through the calculation and simulation, the minimum of thermal resistance has reached $0.019 \mathrm{~W} /{ }^{\circ} \mathrm{C}$.

\section{References}

1. Tuckerman DB, Pease RFW (1981) High performance heat sinking for VLSI. IEEE Electron Device Lett 2(5):126-129

2. Liu L, Zhang GQ, Yang DG, Pan KL, Zhong H, Hou FZ (2010) Thermal analysis and comparison of heat dissipation methods on high-power LEDs. In: IEEE high density packaging

3. Li RC (2010) Research on thermal resistance of high-power LED arrays based on microchannel cooler. Semicond Optoelectron 31(4):8

4. Mao ZM (2012) Thermal modeling and design for microchannel cold plate with high temperature uniformity subjected to multiple heat sources. Int Commun Heat Mass Transf 39(6):781-785

5. Ge J, He W (2008) Thermal analysis and simulation for hydrocooling-heat sink of transistors. Mach Tool Hydraul (5):161-164

6. Knight RW, Hall DJ, Goodling JS, Jaeger RC (1992) Heat sink optimization with application to microchannels. IEEE Trans Compon Hybrids Manuf Technol 15(5):832-842

7. Lee PS, Garimella SV (2006) Thermally developing flow and heat transfer in rectangular microchannels of different aspect ratios. Int J Heat Mass Transf 49:3060-3067

8. Yuan LL, Liu S, Chen MX, Luo XB (2006) Thermal analysis of high-power LED array packaging with microchannel cooler. In: 7th IEEE international conference on electronics packaging technology, 26-29 August 2006

9. Ma ZT, Wang XJ, Zhu DQ, Liu S (2005) Thermal analysis and modeling of LED arrays integrated with an innovative liquidcooling module. In: 6th International conference on electronic packaging technology, Shenzhen, 2-2 September 2005

10. Kishimoto T, Ohsaki T (1986) VLSI packaging technique using liquid-cooled channels. IEEE Trans Compon Hybrids Manuf Technol 9(4):328-335

11. Yang CC, Wang CQ, Hang CJ (2010) Water cooling design for high-power LED multi-chip module. Electron Process Technol 31(5):253-257

12. Steinke ME, Kandlikar SG (2004) Single-phase heat transfer enhancement techniques in microchannel and minichannel flows. In: Proceedings of the 2nd international conference on microchannels and minichannels

13. Shi S, Li S, Zhang HY (2010) Thermal field calculation and analysis of water-cooled heat sink in a three-level SFC. J Chin Soc Power Eng 30:76-80 